\title{
Unacylated Ghrelin (UnAG): A New Treatment Option for Peripheral Arterial Disease?
}

Gabriele Togliatto, Matteo Traversa, Alberto Orsello and Maria Felice Brizzi*

Department of Medical Sciences, University of Torino, Italy

"Corresponding author: Maria Felice Brizzi, Department of Medical Sciences, University of Torino, Corso Dogliotti 14, 10126, Torino, Italy, Tel: 0116706653; Fax: 011663475; E-mail: mariafelice.brizzi@unito.it

Received date: 24 March 2014, Accepted date: 26 June 2014, Published date: 20 July 2014

Copyright: () 2014 Brizzi MF, et al. This is an open-access article distributed under the terms of the Creative Commons Attribution License, which permits unrestricted use, distribution, and reproduction in any medium, provided the original author(s) and source are credited.

Keywords: Diabetes; PAD; ROS; miRNAs; UnAG; SOD-2

\section{Unacylated Ghrelin (UnAG)}

Peripheral arterial disease (PAD) is a major clinical problem and a significant health care cost worldwide [1]. PAD is the result of a progressive occlusion of the peripheral arteries driven by atherosclerosis [2]. This, in turn, leads to a gradual reduction of blood supply to the limb and the occurrence of symptoms ranging from intermittent claudication to critical limb ischemia [3]. Moreover, as PAD remains under-diagnosed for many years, muscle damage and ulceration are commonly found. PAD is a common vascular complication in diabetic individuals [4] and its incidence and prevalence are expected to increase due to the spread of diabetes and an aging population [5]. In particular, diabetic patients have a high risk of developing PAD which is more severe and diffuse than in nondiabetic patients [4]. Moreover, the presence of PAD in these patients is associated with an increased morbidity and mortality for cardiovascular diseases [1,4]. The impaired blood supply to the ischemic limb and the persistence of an oxidative environment are crucial determinants of skeletal muscle damage in PAD [6]. This is particularly true for diabetic patients with PAD where the impaired oxygen availability further increases the number of dysfunctional mitochondria [6,7] ultimately resulting in a vicious cycle. Indeed, it has been reported that superoxide-dismutase-2 (SOD-2), the primary enzymatic antioxidant defence against ROS production, is deficient in human PAD muscles [6,8]. This implies that targeting ROS generation or improving the initial line of defence against oxidative stress might support cellular functions, thus resulting in improved long-term outcomes. As a proof of concept, antioxidant administration recovers mitochondrial dysfunction and muscle function in humans [9]. So far, surgical or endovascular procedures are considered the primary treatment options commonly approved for PAD patients with serious functional impairments [10]. Early PAD diagnosis and timely revascularization is crucial to limb salvage and improved patient outcomes $[1,10]$. However, even if successful, the majority of patients, particularly diabetics, complain of recurrent symptoms [11]. This implies that, in diabetics and in an aging population with an increasingly high incidence of diabetes, new therapeutic options for PAD are needed [12] Therapeutic options, mainly based on angiogenic growth factor delivery or stem cell-based therapy, have been proposed [13-15]. However, data obtained in clinical trials with diabetic patients are still inconclusive $[4,10,16-18]$. The incomplete response of targeted therapies has driven the development of alternative strategies. Thus far, particular attention has been devoted to develop and test the clinical impact of new therapeutics able to interfere with ROS generation or improving the anti-oxidant machinery [19]. Based on the above considerations, we have recently developed a therapeutic protocol targeting ROS generation and tested its efficacy in a preclinical model of PAD [20]. The protocol was based on the use of the unacylated ghrelin (UnAG). Ghrelin is a small peptide predominantly produced by the stomach [21] that circulates in two different forms: the acylated (AG) and unacylated (UnAG) form [21]. UnAG represents the most abundant circulating ghrelin form [22]. Unlike AG, UnAG does not bind to the Gq-coupled growth hormone (GH) secretagogue receptor type 1a (GHSR1a) [23], thus lacking AG neuroendocrine activities [22]. While both UnAG and AG protect vascular cell from apoptotic signals through a common receptor [24], they exert distinctly different biological actions in neonatal cardiomyocytes [25]. UnAG, but not AG, protects endothelial progenitor cells (EPCs) from ROS-mediated damage in diabetic setting $[26,27]$. We have previously demonstrated that UnAG, unlike AG, by regulating Rac1, prevents senescence and was able to revert diabetesassociated EPC functional impairment [26]. Moreover, we demonstrated that only in diabetic patients UnAG, by restoring eNOS activity via Akt-mediated phosphorylation, improves EPC bone marrow mobilization resulting in a strong increase in the number of circulating cells [26]. Besides acting on vascular cells, UnAG is also able to protect muscles from ischemia-induced tissue damage [20]. Following ischemia, a population of resident stem cells, the satellite cells (SCs), move from quiescence and undergo proliferation to regenerate the damaged muscle [28]. A precise transcriptional regulation involving $\mathrm{MyoD}$ and the myogenic regulatory factor Myf5 controls SC proliferation/differentiation [29]. We demonstrated that UnAG promotes SC proliferation and terminal differentiation in mice subjected to hind-limb ischemia that result in a low damage score. In particular, we found that UnAG administration increases the number of Pax-7+/MyoD+ cells in the ischemic muscles [20]. Moreover, we found that the protection against tissue damage exerted by UnAG mainly depends on its effect on SOD-2 expression. As a proof of concept the formation of TBARS was significantly lower in muscles recovered from UnAG-treated mice compared to those from AG and saline mice [20]. Consistently, ex-vivo experiments performed in SCs isolated from mice subjected to UnAG treatment, displayed lower intracellular ROS content and higher SOD-2 protein expression compared to control animals [20]. Again, as a proof of concept, silencing SOD-2 prevents UnAG-mediated SC proliferation/ differentiation upon ischemia. Skeletal muscle regeneration is under the control of post-transcriptional mechanisms mainly involving small-non coding microRNAs (miRs) [30-32]. Recently different families of miRs have attracted particular interest, and among them miR221/222 [33,34]. At this regard we found that, besides regulating endothelial cell fate [35,36], miR221/222 are involved in UnAGinduced skeletal muscle regeneration following ischemia. In particular, we demonstrated that the expression of miR-221/222 was significantly increased in SCs recovered from muscles of UnAG-treated mice [20]. 
Page 2 of 3

The Cip/Kip family members p27Kip1 and p57Kip2 are known to be regulated by miR221/222 in different pathological contexts [35,36]. Likewise, we found that p57Kip2, but not p27Kip1, is posttranscriptionally controlled by miR221/222 upon UnAG treatment. The role of miR221/222 in mediating UnAG action in this particular pathological setting was further validated by in vivo gain-of-function experiments. Indeed, we found that miR221/222 administration recapitulates skeletal muscle regeneration upon ischemia even in the absence of UnAG. Of interest siRNA technology led us to demonstrate the crucial role of SOD-2 in the control of miR221/222 expression following ischemia (Figure 1) [20].

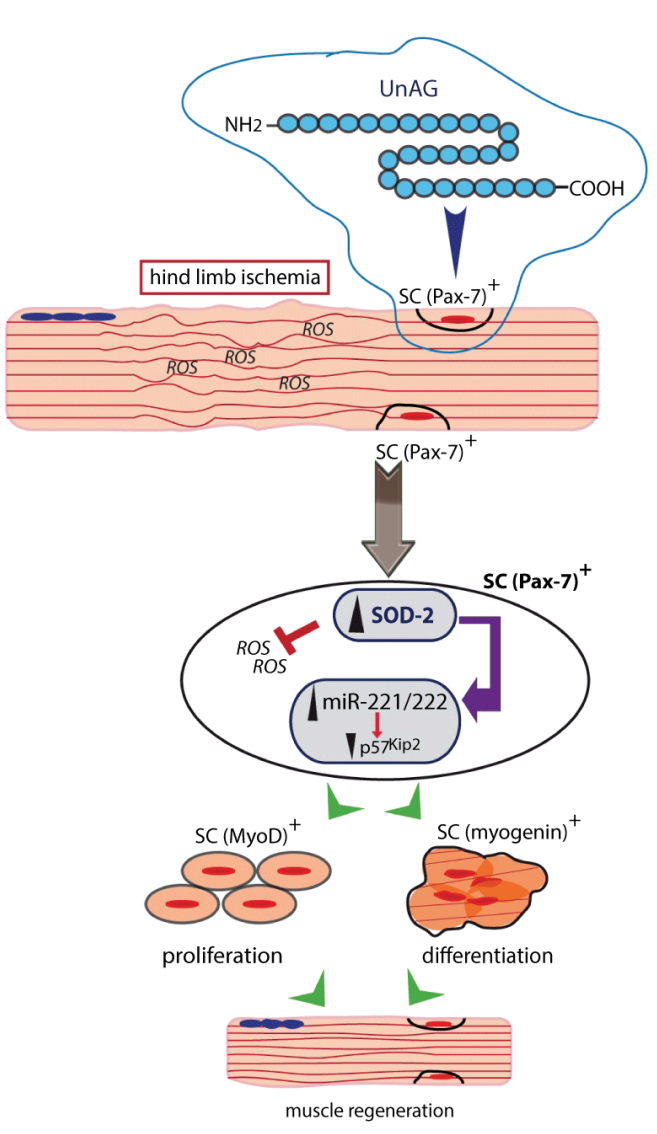

Figure 1: Schematic representation of the molecular events challenged by UnAG and involved in skeletal muscle regeneration upon ischemia. Following muscle damage quiescent SCs undergo proliferation as demonstrated by the number of Pax7/MyoD+ cells in muscle subjected to UnAG treatment. This effect is mediated by SOD-2 expression and miR-221/222-driven p57Kip2 posttranscriptional regulation. As the result of SOD-2-mediated ROS protection UnAG also contributes to SC differentiation (revealed by the increased number of myogenin+ cells) and myofiber regeneration. SC: Satellite Cell; UnAG: Unacylated Ghrelin; SOD-2: Superoxide Dismutase-2; ROS: Reactive Oxygen Species; miR: miRNAs.

At present the number of patients eligible for revascularization are negligible and current surgical or endovascular approaches are associated with increased perioperative complications [10]. This is particularly true in diabetic individuals for whom, in many cases, amputation remains the main option. This implies that alternative therapeutic approaches, based on the use of naturally occurring molecules able to protect muscle from ROS-mediated damage, might represent a valuable choice. In addition, as UnAG specifically improves the efficacy of the anti-oxidant machinery, further efforts should be directed to test its clinical benefits in pathological conditions denoted by ROS-mediated damage.

\section{Acknowledgments}

This work was supported by grants obtained by MFB from UnitoCompagnia S. Paolo and from Fondazione per la Ricerca Diabetologica FO.Ri.SID.

\section{References}

1. Mahoney EM, Wang K, Keo HH, Duval S, Smolderen KG, et al. (2010) Vascular hospitalization rates and costs in patients with peripheral artery disease in the United States. Circ Cardiovasc Qual Outcomes 3: 642-651.

2. Norgren L, Hiatt WR, Dormandy JA, Nehler MR, Harris KA, et al. (2007) Inter-Society Consensus for the Management of Peripheral Arterial Disease (TASC II). J Vasc Surg 45 Suppl S: S5-67.

3. Gray BH, Conte MS, Dake MD, Jaff MR, Kandarpa K, et al. (2008) Atherosclerotic Peripheral Vascular Disease Symposium II: lowerextremity revascularization: state of the art. Circulation 118: 2864-2872.

4. American Diabetes Association (2003) Peripheral arterial disease in people with diabetes. Diabetes Care 26: 3333-3341.

5. Beckman JA, Paneni F, Cosentino F, Creager MA (2013) Diabetes and vascular disease: pathophysiology, clinical consequences, and medical therapy: part II. Eur Heart J 34: 2444-2452.

6. Pipinos II, Judge AR, Selsby JT, Zhu Z, Swanson SA, et al. (2008) The myopathy of peripheral arterial occlusive disease: Part 2. Oxidative stress, neuropathy, and shift in muscle fiber type. Vasc Endovascular Surg 42: 101-112.

7. Bhat HK, Hiatt WR, Hoppel CL, Brass EP (1999) Skeletal muscle mitochondrial DNA injury in patients with unilateral peripheral arterial disease. Circulation 99: 807-812.

8. Makris KI, Nella AA, Zhu Z, Swanson SA, Casale GP, et al. (2007) Mitochondriopathy of peripheral arterial disease. Vascular 15: 336-343.

9. Loffredo L, Marcoccia A, Pignatelli P, Andreozzi P, Borgia MC, et al. (2007) Oxidative-stress-mediated arterial dysfunction in patients with peripheral arterial disease. Eur Heart J 28: 608-612.

10. Weinberg MD, Lau JF, Rosenfield K, Olin JW (2011) Peripheral artery disease. Part 2: medical and endovascular treatment. Nat Rev Cardiol 8: 429-441.

11. Cieri E, Lenti M, De Rango P, Isernia G, Marucchini A, et al. (2011) Functional ability in patients with critical limb ischaemia is unaffected by successful revascularisation. Eur J Vasc Endovasc Surg 41: 256-263.

12. Sneider EB, Nowicki PT, Messina LM (2009) Regenerative medicine in the treatment of peripheral arterial disease. J Cell Biochem 108: 753-761.

13. Jones WS, Annex BH (2007) Growth factors for therapeutic angiogenesis in peripheral arterial disease. Curr Opin Cardiol 22: 458-463.

14. Yan J, Tie G, Xu TY, Cecchini K, Messina LM (2013) Mesenchymal stem cells as a treatment for peripheral arterial disease: current status and potential impact of type II diabetes on their therapeutic efficacy. Stem Cell Rev 9: 360-372.

15. Raval Z, Losordo DW (2013) Cell therapy of peripheral arterial disease: from experimental findings to clinical trials. Circ Res 112: 1288-1302.

16. Weitz J, Byrne J, Clagett GP, Farkouh ME, Porter JM, et al. (1996) Diagnosis and treatment of chronic arterial insufficiency of the lower extremities: a critical review. Circulation 94: 3026-3049.

17. Gili M, Orsello A, Gallo S, Brizzi MF (2013) Diabetes-associated macrovascular complications: cell-based therapy a new tool? Endocrine 44: 557-575. 
Citation: Togliatto G, Traversa M, Orsello A, Brizzi MF (2014) Unacylated Ghrelin (UnAG): A New Treatment Option for Peripheral Arterial Disease?. J Mol Genet Med 8: 108. doi:10.4172/1747-0862.1000108

Page 3 of 3

18. Sara Gallo, Maddalena Gili, Gabriele Togliatto, Maria Felice Brizzi. Diabetes-associated vascular complications: mechanisms of disease progression and alternative therapeutic options Journal of Molecular and Genetic Medicine. In press

19. Pipinos II, Judge AR, Zhu Z, Selsby JT, Swanson SA, et al. (2006) Mitochondrial defects and oxidative damage in patients with peripheral arterial disease. Free Radic Biol Med 41: 262-269.

20. Togliatto G, Trombetta A, Dentelli P, Cotogni P, Rosso A, et al. (2013) Unacylated ghrelin promotes skeletal muscle regeneration following hindlimb ischemia via SOD-2-mediated miR-221/222 expression. J Am Heart Assoc 2: e000376.

21. Kojima M, Hosoda H, Date Y, Nakazato M, Matsuo H, et al. (1999) Ghrelin is a growth-hormone-releasing acylated peptide from stomach. Nature 402: 656-660.

22. van der Lely AJ, Tschöp M, Heiman ML, Ghigo E (2004) Biological, physiological, pathophysiological, and pharmacological aspects of ghrelin. Endocr Rev 25: 426-457.

23. Howard AD, Feighner SD, Cully DF, Arena JP, Liberator PA, et al. (1996) A receptor in pituitary and hypothalamus that functions in growth hormone release. Science 273: 974-977.

24. Baldanzi G, Filigheddu N, Cutrupi S, Catapano F, Bonissoni S, et al (2002) Ghrelin and des-acyl ghrelin inhibit cell death in cardiomyocytes and endothelial cells through ERK1/2 and PI 3-kinase/AKT. J Cell Biol 159: 1029-1037.

25. Lear PV, Iglesias MJ, Feijóo-Bandín S, Rodríguez-Penas D, MosqueraLeal A, et al. (2010) Des-acyl ghrelin has specific binding sites and different metabolic effects from ghrelin in cardiomyocytes. Endocrinology 151: 3286-3298.

26. Togliatto G, Trombetta A, Dentelli P, Baragli A, Rosso A, et al. (2010) Unacylated ghrelin rescues endothelial progenitor cell function in individuals with type 2 diabetes. Diabetes 59: 1016-1025.
27. Granata R, Settanni F, Julien M, Nano R, Togliatto G, et al. (2012) Desacyl ghrelin fragments and analogues promote survival of pancreatic Bcells and human pancreatic islets and prevent diabetes in streptozotocintreated rats. J Med Chem 55: 2585-2596.

28. Kuang S, Gillespie MA, Rudnicki MA (2008) Niche regulation of muscle satellite cell self-renewal and differentiation. Cell Stem Cell 2: 22-31.

29. Yin H, Price F, Rudnicki MA (2013) Satellite cells and the muscle stem cell niche. Physiol Rev 93: 23-67.

30. Rao PK, Kumar RM, Farkhondeh M, Baskerville S, Lodish HF (2006) Myogenic factors that regulate expression of muscle-specific microRNAs. Proc Natl Acad Sci U S A 103: 8721-8726.

31. Chen JF, Mandel EM, Thomson JM, Wu Q, Callis TE, et al. (2006) The role of microRNA-1 and microRNA-133 in skeletal muscle proliferation and differentiation. Nat Genet 38: 228-233.

32. Sweetman D, Goljanek K, Rathjen T, Oustanina S, Braun T, et al. (2008) Specific requirements of MRFs for the expression of muscle specific microRNAs, miR-1, miR-206 and miR-133. Dev Biol 321: 491-499.

33. Cardinali B, Castellani L, Fasanaro P, Basso A, Alemà S, et al. (2009) Microrna-221 and microrna-222 modulate differentiation and maturation of skeletal muscle cells. PLoS One 4: e7607.

34. Greco S, Perfetti A, Fasanaro P, Cardani R, Capogrossi MC, et al. (2012) Deregulated microRNAs in myotonic dystrophy type 2. PLoS One 7: e39732.

35. Togliatto G, Trombetta A, Dentelli P, Rosso A, Brizzi MF (2011) MIR221/MIR222-driven post-transcriptional regulation of P27KIP1 and P57KIP2 is crucial for high-glucose- and AGE-mediated vascular cell damage. Diabetologia 54: 1930-1940.

36. Dentelli P, Rosso A, Orso F, Olgasi C, Taverna D, et al. (2010) microRNA-222 controls neovascularization by regulating signal transducer and activator of transcription 5A expression. Arterioscler Thromb Vasc Biol 30: 1562-1568. 